\title{
Effect of Nitrogen and Potassium on Growth, Yield, and Seed Quality of Quinoa in Ferralsols and Acrisols under Rainfed Conditions
}

\author{
Nguyen Van Minh'1, Dinh Thai Hoang ${ }^{2}$, Dang Thi Phuong Anh'², Nguyen Viet Long² \\ 1 Faculty of Agriculture and Forestry, Tay Nguyen University, Dak Lak, 567 Le Duan Street, Buon Ma Thuot City, \\ Dak Lak province, 63100, Vietnam \\ 2 Faculty of Agronomy, Vietnam National University of Agriculture, Hanoi, 131000, Vietnam \\ * Corresponding author's e-mail: nvlong@vnua.edu.vn
}

\begin{abstract}
The study has investigated the individual and combined effects of nitrogen and potassium on the growth, grain yield, and quality of quinoa in ferralsols and acrisols. The experiments were conducted during the dry season under rainfed conditions in Central Highland, Vietnam. The factorial design was a randomized complete block design with three replications. The results revealed the positive impacts of nitrogen and potassium on the growth and yield of quinoa. However, after the application of an optimum dose of nitrogen, growth, and yield were not significantly changed and even decreased when the dose continued increasing. Higher levels of nitrogen and potassium application resulted in greater protein and fat content, but lower starch and fiber contents, compared to lower levels. The fertilizer practice has to rely on soil fertility. The study shows that the application of $150 \mathrm{~kg} \mathrm{~N}$ and $105 \mathrm{~kg} \mathrm{~K}_{2} \mathrm{O} \mathrm{ha}{ }^{-1}$ could be the optimum rate of nitrogen and potassium for quinoa production in ferralsols and acrisols in Central Highland.
\end{abstract}

Keywords: acrisols, ferralsols, nitrogen, potassium, quinoa.

\section{INTRODUCTION}

Quinoa (Chenopodium quinoa Willd.), an ancient staple food crop, recently has been spread for growing worldwide. It could flourish in various agro-ecological zones with a wide range of adaptation of relative humidities (40-80\%), temperatures $\left(-4\right.$ to $\left.38^{\circ} \mathrm{C}\right)$, being highly tolerant to soil moisture deficiency. In addition, quinoa is considered a high nutritional plant food that provides a high content of protein with all essential amino acids, fats, carbohydrates, minerals, and vitamins, as well as possesses nutraceutical and medicinal properties (FAO, 2011). In order for quinoa to grow and develop well and to reach high yield and high seed quality, it requires sufficient amount of essential nutrients, especially nitrogen $(\mathrm{N})$ and potassium $(\mathrm{K})$ (Alvar-Beltrán et al., 2021). Numerous studies demonstrated the role of $\mathrm{N}$ in enhancing the growth, yield, and seed quality of quinoa (Jacobsen et al., 1994;
Thanapornpoonpong, 2004; Kaul et al., 2005; Abou-Amer and Kamel, 2011; Gomaa, 2013; Basra et al., 2014; Kakabouki et al., 2014; Geren, 2015; Fawy et al., 2017; Almadini et al., 2019; Owji et al., 2020; Wang et al., 2020; Al-asadi et al., 2021). Meanwhile, limited information has been reported on the effects of $\mathrm{K}$ on quinoa growth and yield (Rêgo et al., 2017; Salim et al., 2019; Turcios et al., 2020). Moreover, nutrient balance is a key factor to promote plant growth and yield. Hou et al. (2018) demonstrated that the imbalance between $\mathrm{N}$ and $\mathrm{K}$ might lead to the yield reduction in a cereal crop. However, the information on the balance of $\mathrm{N}$ and $\mathrm{K}$ in quinoa has been also not clear.

Climate change with the phenomenon of raising drought status is one of the factors that constrain agricultural production. According to the World Bank's forecast (2016), agricultural production will be soon less effective because over $70 \%$ of the production area is now subject 
to rain-fed conditions. Vietnam is ranked in the top of the five most-affected countries by climate risk with extreme droughts continued well and recorded as the worst droughts in the last 100 years (Eckstein et al., 2017). Quinoa was first evaluated to grow well in Vietnam in 1986 with a higher potential yield than those in native regions (Trinh, 2001; Betero et al., 2004). Recently, quinoa adapted to grow in difficult cultivation regions where agricultural production is subjected to drought or salinity stress in the whole country (Nguyen, 2020). Central Highland, with a relatively flat and large topography is considered a favorable region for developing quinoa production. In this region, ferralsols and acrisols soils are two typical soils that account for 24.09 and $64.43 \%$ of total natural land. Quinoa was demonstrated to grow well in ferralsols soils in this region (Nguyen et al., 2020). However, this kind of soil is also suitable for perennial crops, such as coffee, rubber, or pepper. Although drought stress constrains the production, these crops still contributed substantially to Vietnam's agricultural exports. Therefore, the potential for growing quinoa in acrisols soil should be discovered.
Our main objectives were to investigate the individual and combined effects of $\mathrm{N}$ and $\mathrm{K}$ on the growth, yield, and quality of quinoa under both ferralsols and acrisols soils conditions in rain-fed areas; and to recommend the optimum of $\mathrm{N}$ and $\mathrm{K}$ doses to fertilize quinoa under such conditions.

\section{MATERIALS AND METHODS}

\section{Plant materials}

The quinoa variety Atlas introduced from the Netherlands was used in the performed research. This was recommended as drought tolerance (Nguyen et al., 2021) and the best adaptive variety in Central Highland (Nguyen et al., 2020).

\section{Experimental conditions}

The experiments were conducted in the dry season from February to May 2021 at two locations in the Central Highland region, Vietnam. The weather conditions were shown in Table 1 with total rainfall of $357.2 \mathrm{~mm}$ and $144.4 \mathrm{~mm}$ in Dak Lak and Dak Nong province, respectively. The experimental soils were typical soil in

Table 1. Soil physicochemical properties

\begin{tabular}{|c|c|c|}
\hline Parameters & Ferralsols soil in Dak Lak & Acrisols soil in Dak Nong \\
\hline $\mathrm{pH}$ & 5.08 & 4.71 \\
\hline OM (\%) & 4.36 & 2.44 \\
\hline Total N & 0.19 & 0.09 \\
\hline Total P & 0.21 & 0.11 \\
\hline Total K & 0.15 & 0.08 \\
\hline Available P $\left({\left.\mathrm{mg} 100 \mathrm{~g}^{-1}\right)}\right.$ & 8.07 & 2.74 \\
\hline Available K (mg 100g $\left.{ }^{-1}\right)$ & 20.84 & 7.01 \\
\hline Exchangeable Ca (meq $100 \mathrm{~g}^{-1}$ ) & 2.78 & 0.56 \\
\hline Exchangeable Mg (meq $\left.100 \mathrm{~g}^{-1}\right)$ & 2.17 & 0.11 \\
\hline
\end{tabular}

Table 2. Meteorological Records for Dak Lak and Dak Nong provinces in 2020

\begin{tabular}{|l|c|c|c|c|c|c|c|c|}
\hline \multirow{2}{*}{ Factors } & \multicolumn{4}{|c|}{ Dak Lak } & \multicolumn{4}{c|}{ Dak Nong } \\
\cline { 2 - 10 } & Feb & Mar & Apr & May & Feb & Mar & Apr & May \\
\hline Average temp. $\left({ }^{\circ} \mathrm{C}\right)$ & 23.5 & 24.1 & 25.0 & 25.7 & 22.7 & 26.3 & 26.7 & 28.0 \\
\hline Max temp. $\left({ }^{\circ} \mathrm{C}\right)$ & 35.0 & 34.3 & 34.0 & 33.5 & 26.6 & 28.3 & 28.6 & 31.9 \\
\hline Min temp. $\left({ }^{\circ} \mathrm{C}\right)$ & 18.0 & 18.1 & 18.5 & 21.5 & 20.4 & 24.7 & 23.3 & 25.1 \\
\hline Rainfall $(\mathrm{mm})$ & 5.0 & 33.1 & 115.0 & 204.1 & 0 & 0 & 34.6 & 109.8 \\
\hline Rain days (day) & 1 & 2 & 4 & 22 & 0 & 0 & 3 & 11 \\
\hline Humidity (\%) & 68 & 74 & 78 & 83 & 70 & 68 & 70 & 73 \\
\hline Total sunny (hours) & 280 & 264 & 245 & 206.5 & 266.0 & 298.8 & 265.4 & 241.9 \\
\hline
\end{tabular}

Source: Hydrometeorological Center of Dak Lak and Dak Nong Provinces (2020) 
Central Highland: a grey soil in Dak Nong with lower $\mathrm{pH}$ and poorer soil properties compared to a red basalt soil in Dak Lak (Table 2).

\section{Experimental design}

The experimental design was a randomized complete block design (RCBD) with three replications. The distance between each replication was $1 \mathrm{~m}$. The plot area was $14 \mathrm{~m}^{2}(5 \times 2.8 \mathrm{~m})$. The experimental factors included four $\mathrm{N}$ levels $(60$, 90,120 , and $\left.150 \mathrm{~kg} \mathrm{~N} \mathrm{ha}^{-1}\right)$ and four $\mathrm{K}$ levels (60, 75, 90, and $105 \mathrm{~kg} \mathrm{~K}_{2} \mathrm{O} \mathrm{ha}^{-1}$ ).

\section{Crop management}

The experimental land was plowed and raked carefully at depth of 20 to $30 \mathrm{~cm}$. Weeds were cleared before sowing. Seeds were sowed in rows with a distance of $50 \times 30 \mathrm{~cm}$ at $2-3 \mathrm{~cm}$ of depths, then thinned to one plant per hill at the 2-3 leaves stage. The basal fertilizer was applied before sowing with an amount of 1 ton Huco microbial organic fertilizer, $500 \mathrm{~kg}$ lime, and $60 \mathrm{~kg} \mathrm{P}_{2} \mathrm{O}_{5}$ (superphosphate). Urea $(46 \% \mathrm{~N})$ and potassium chloride $\left(60 \% \mathrm{~K}_{2} \mathrm{O}\right)$ were used for top dressing at 20 and 40 days after sowing. The quinoa plants grew under rain-fed conditions with water supplements at the sowing date to ensure seed germination. Crop management was regularly practiced to control pests, diseases, and weeds.

\section{Data collection}

At harvest, the 10 sample plants were randomly selected from each experimental plot to measure plant height, stem diameter, the number of branches, the number of panicles, and the number of seeds per panicle. The harvested seeds were sun-dried for 3 days to determine 1000-seed weight and plot yield. The seed sample then was oven-dried at $60^{\circ} \mathrm{C}$ until constant weight to determine the contents of protein, starch, fat, fiber, and ash contents according to the methods described in detail by Eisa et al. (2018).

\section{Data analysis}

The data were subjected to analysis of variance according to a randomized complete block design for a factorial experiment using Minitab 16. Grouping information using the Tukey method was done with a confidence of $95.0 \%$.

\section{RESULTS}

\section{Effect of nitrogen and potassium on growth characteristics of quinoa}

Different $\mathrm{N}$ and $\mathrm{K}$ application levels had significant effects on plant height, plant diameter, and the number of branches at both locations (except for the effect of $\mathrm{K}$ on the number of branches in Dak Nong). In general, there were upward trends in all investigated parameters when increasing the $\mathrm{N}$ and $\mathrm{K}$ application levels (Table 3). In fact, applying $\mathrm{N}$ at $150 \mathrm{~kg} \mathrm{~N} \mathrm{ha}^{-1}$ resulted in significantly higher plant height and the number of branches compared to the lowest $\mathrm{N}$ levels (60 and $90 \mathrm{~kg} \mathrm{~N} \mathrm{ha}^{-1}$ ). At $120 \mathrm{~kg} \mathrm{~N}^{-}$ ${ }^{1}$, the number of branches was highest, significantly higher than those at 60 and $90 \mathrm{~kg} \mathrm{~N} \mathrm{ha}^{-1}$. The application of $\mathrm{K}$ at the rate of $105 \mathrm{~kg} \mathrm{~K}_{2} \mathrm{O}$ $\mathrm{ha}^{-1}$ achieved better values for all parameters. However, in Dak Lak it resulted in significantly higher values than those at rates of 60 and $75 \mathrm{~kg}$ $\mathrm{K}_{2} \mathrm{O} \mathrm{ha} \mathrm{h}^{-1}$ for plant diameter and $60 \mathrm{~kg} \mathrm{~K}_{2} \mathrm{O}$ for the number of branches only. In Dak Nong, it resulted in significantly higher values than those at rates of $60 \mathrm{~kg} \mathrm{~K}_{2} \mathrm{O} \mathrm{ha}{ }^{-1}$ for plant height and 60 and $75 \mathrm{~kg} \mathrm{~K}_{2} \mathrm{O} \mathrm{ha}^{-1}$ for plant diameter only. The interaction of $\mathrm{N}$ and $\mathrm{K}$ was significant for most parameters, except for plant diameter and the number of branches in Dak Lak (Table 3). Applying $\mathrm{N}$ and $\mathrm{K}$ at rates of $90 \mathrm{~kg} \mathrm{~K}_{2} \mathrm{O}$ combined with 120 and $150 \mathrm{~kg} \mathrm{~N} \mathrm{ha}^{-1}$ seemed to be better for quinoa growth than other combinations.

\section{Effect of nitrogen and potassium on yield components of quinoa}

$\mathrm{N}$ application had noticeable impact on yield components including the number of main panicles, the number of seeds per panicle, 1000-seed weight, and seed yield of quinoa, except for the number of seeds per panicle in Dak Nong (Table 4).

In Dak Lak, yield components increased along with the rate of $\mathrm{N}$ application, then decreased after reaching a peak at $120 \mathrm{~kg} \mathrm{~N} \mathrm{ha}^{-1}$. The clearest trend was observed in seed yield with the highest value of 23.0 ton ha ${ }^{-1}$. However, significant differences were only found among $\mathrm{N}$ treatments for seed yield, and between lower $(60$ and $90 \mathrm{~kg} \mathrm{~N}$ $\left.\mathrm{ha}^{-1}\right)$ and higher rate treatments (120 and $150 \mathrm{~kg}$ $\mathrm{N} \mathrm{ha}^{-1}$ ) for all other traits. In Dak Nong, higher values for yield components were the result of 
Table 3. Effects of nitrogen and potassium on plant height (PH), plant diameter (PD), the number of branches (No.B) of quinoa in Dak Lak and Dak Nong provinces

\begin{tabular}{|c|c|c|c|c|c|c|c|}
\hline \multirow{2}{*}{\multicolumn{2}{|c|}{ Treatment }} & \multicolumn{3}{|c|}{ Dak Lak } & \multicolumn{3}{|c|}{ Dak Nong } \\
\hline & & $\mathrm{PH}$ & PD & No.B & $\mathrm{PH}$ & PD & No.B \\
\hline \multirow{4}{*}{$\mathrm{N}$} & 60 & $128.8 \mathrm{~B}$ & $13.1 \mathrm{C}$ & $26.6 \mathrm{~B}$ & $115.7 \mathrm{C}$ & $13.6 \mathrm{C}$ & $27.0 \mathrm{~B}$ \\
\hline & 90 & 129.1B & $13.3 \mathrm{BC}$ & 28.5B & $118.8 \mathrm{BC}$ & $14.6 \mathrm{BC}$ & $28.6 \mathrm{AB}$ \\
\hline & 120 & $147.1 \mathrm{~A}$ & $19.2 \mathrm{~A}$ & 33.7AB & 126.2AB & 16.7AB & $30.2 A B$ \\
\hline & 150 & $151.7 \mathrm{~A}$ & $15.8 \mathrm{~B}$ & $30.2 \mathrm{~A}$ & $130.1 \mathrm{~A}$ & $15.8 \mathrm{~A}$ & $32.2 \mathrm{~A}$ \\
\hline \multirow{4}{*}{$\mathrm{K}$} & 60 & $135.4 \mathrm{~B}$ & $13.5 \mathrm{~B}$ & $27.6 \mathrm{~B}$ & $117.5 \mathrm{~B}$ & $13.5 \mathrm{C}$ & $28.6 \mathrm{~A}$ \\
\hline & 90 & 138.1B & $14.5 \mathrm{~B}$ & $29.2 A B$ & $120.7 \mathrm{AB}$ & 14.6BC & $29.1 \mathrm{~A}$ \\
\hline & 120 & $137.0 \mathrm{~B}$ & $15.8 \mathrm{AB}$ & $30.3 \mathrm{AB}$ & 123.8AB & $15.8 \mathrm{AB}$ & $30.0 \mathrm{~A}$ \\
\hline & 150 & $146.1 \mathrm{~A}$ & $17.6 \mathrm{~A}$ & $31.9 \mathrm{~A}$ & $128.8 \mathrm{~A}$ & $16.9 \mathrm{~B}$ & $30.4 \mathrm{~A}$ \\
\hline \multirow{16}{*}{$\mathrm{N}: \mathrm{K}$} & $60: 60$ & $129.7 \mathrm{ef}$ & $12.7 b-d$ & $23.4 b$ & $102.4 d$ & $10.3 c$ & $24.9 a$ \\
\hline & $60: 90$ & $135.5 c-f$ & $10.4 d$ & $29.3 a b$ & $121.8 \mathrm{a}-\mathrm{d}$ & $14.1 \mathrm{~b}$ & $25.3 a$ \\
\hline & $60: 120$ & $121.9 f$ & $14.6 b-d$ & $27.2 a b$ & $124.2 \mathrm{a}-\mathrm{d}$ & $14.8 \mathrm{~b}$ & $25.6 a$ \\
\hline & $60: 150$ & $128.1 \mathrm{ef}$ & $14.5 b-d$ & $26.6 a b$ & 114.5a-d & $15.1 b$ & $32.1 \mathrm{a}$ \\
\hline & $90: 60$ & 129.1ef & $11.4 \mathrm{~cd}$ & $28.0 \mathrm{ab}$ & $123.3 \mathrm{a}-\mathrm{d}$ & $14.1 \mathrm{~b}$ & $40.0 a$ \\
\hline & $90: 90$ & $132.1 \mathrm{~d}-\mathrm{f}$ & $13.9 \mathrm{~cd}$ & 28.7ab & $111.0 \mathrm{a}-\mathrm{d}$ & $14.7 \mathrm{~b}$ & $26.8 a$ \\
\hline & $90: 120$ & 121.0ef & $13.4 b-d$ & $29.4 a b$ & $119.0 \mathrm{a}-\mathrm{d}$ & $14.7 \mathrm{~b}$ & $27.7 a$ \\
\hline & $90: 150$ & $134.1 d-f$ & $14.6 b-d$ & $27.7 a b$ & $122.0 \mathrm{a}-\mathrm{d}$ & $14.9 b$ & $30.0 a$ \\
\hline & $120: 60$ & 133.2d-f & $15.6 b-d$ & $32.1 \mathrm{ab}$ & $119.3 a-d$ & $15.0 \mathrm{~b}$ & $30.0 \mathrm{a}$ \\
\hline & $120: 90$ & $141.8 \mathrm{~b}-\mathrm{e}$ & $18.1 \mathrm{a}-\mathrm{c}$ & $30.7 a b$ & $123.0 \mathrm{a}-\mathrm{d}$ & $14.6 \mathrm{~b}$ & $32.0 \mathrm{a}$ \\
\hline & $120: 120$ & 150.7a-d & 19.7ab & $35.7 a b$ & $122.4 a-d$ & $18.5 a$ & $33.2 a$ \\
\hline & $120: 150$ & $162.7 a$ & $23.3 a$ & $36.4 a$ & $140.0 a$ & $18.8 a$ & $25.7 a$ \\
\hline & $150: 60$ & $149.7 a-d$ & $14.1 \mathrm{~b}-\mathrm{d}$ & $27.1 \mathrm{ab}$ & 125.1a-d & $14.4 \mathrm{~b}$ & $29.4 a$ \\
\hline & $150: 90$ & $142.9 b-e$ & $15.5 b-d$ & $28.0 a b$ & $126.9 a-d$ & $14.9 \mathrm{~b}$ & $32.4 a$ \\
\hline & $150: 120$ & $154.5 \mathrm{a}-\mathrm{c}$ & $15.6 b-d$ & $29.0 \mathrm{ab}$ & $129.7 a-c$ & $15.0 \mathrm{~b}$ & $33.3 a$ \\
\hline & $150: 150$ & $159.6 a b$ & 18.0a-c & $36.8 a$ & $138.7 \mathrm{ab}$ & $18.9 a$ & $33.9 \mathrm{a}$ \\
\hline \multicolumn{8}{|c|}{ Analysis of variance } \\
\hline & $\mathrm{N}$ & *** & $* * *$ & $* * \star$ & ** & $* * *$ & ** \\
\hline & $\mathrm{K}$ & ** & ** & * & * & *** & ns \\
\hline & $x \mathrm{~K}$ & ** & ns & ns & * & ** & * \\
\hline
\end{tabular}

Different capital letters show significance among $\mathrm{N}$, and $\mathrm{K}$ treatments, different lowercase letters show significance among $\mathrm{N}$ combined $\mathrm{K}$ treatments at $\mathrm{p}<0.05$ by Turkey's test. $\mathrm{ns}=$ not significant; $*=$ significant at $0.05 ; * *=$ significant at $0.01 ; * * *=$ significant at 0.001 .

increasing $\mathrm{N}$ application from 60 to $150 \mathrm{~kg} \mathrm{ha}^{-1}$. However, significant differences were also found among $\mathrm{N}$ treatments for seed yield, and between the highest rate $\left(150 \mathrm{~kg} \mathrm{~N} \mathrm{ha}^{-1}\right)$ with other treatments for other parameters.

The difference in $\mathrm{K}$ application caused variations in the yield components of quinoa for the number of panicles, the number of seeds per panicle, and seed yield in Dak Lak, and for the number of main panicles and seed yield in Dak Nong (Table 5). There were similar trends with increasing yield components by increasing $\mathrm{K}$ application rates at both locations. In fact, significant differences were found between lower (60 and $75 \mathrm{~kg} \mathrm{~K} 2 \mathrm{O}$ $\mathrm{ha}^{-1}$ ) and higher rates (90 and $105 \mathrm{~kg} \mathrm{~K} \mathrm{O}_{2} \mathrm{ha}^{-1}$ ) for the panicle number, and the seed number per panicle in Dak Lak, and between the rate of 105 $\mathrm{kg} \mathrm{K}_{2} \mathrm{O}_{\mathrm{ha}^{-1}}$ with other $\mathrm{K}$ treatments. Seed yield was highest with 22.9 ton $\mathrm{ha}^{-1}$ in Dak Lak and 17.7 ton ha ${ }^{-1}$ in Dak Nong when $\mathrm{K}$ was applied at the rate of $105 \mathrm{~kg} \mathrm{~K}_{2} \mathrm{O} \mathrm{ha}^{-1}$.

The interaction of $\mathrm{N}$ and $\mathrm{K}$ was significant for panicle number, the number of seeds per panicle in Dak Lak, and seed yield in both locations. The better values for yield components traits seemed to be the results of the combination between $\mathrm{K}$ at the rate of $105 \mathrm{~kg} \mathrm{~K}_{2} \mathrm{O} \mathrm{ha}^{-1}$ with $\mathrm{N}$ at the rates of 120 and $150 \mathrm{~kg} \mathrm{~N} \mathrm{ha}^{-1}$. 
Table 4. Effects of nitrogen and potassium on the number of the main panicle (PH), the number of seeds per the main panicle (No.S), 1000-seed weight (P1000), and seed yield (SY) of quinoa in Dak Lak and Dak Nong provinces

\begin{tabular}{|c|c|c|c|c|c|c|c|c|c|}
\hline \multirow{2}{*}{\multicolumn{2}{|c|}{ Treatment }} & \multicolumn{4}{|c|}{ Dak Lak } & \multicolumn{4}{|c|}{ Dak Nong } \\
\hline & & \multirow{2}{*}{$\frac{\text { No.P }}{37.6 \mathrm{C}}$} & \multirow{2}{*}{$\frac{\text { No.S }}{267.4 \mathrm{~B}}$} & \multirow{2}{*}{$\frac{\mathrm{P} 1000}{3.42 \mathrm{~B}}$} & \multirow{2}{*}{$\frac{S Y}{17.7 D}$} & \multirow{2}{*}{$\frac{\text { No.P }}{31.1 \mathrm{~B}}$} & \multirow{2}{*}{$\frac{\text { No.S }}{249.5 \mathrm{~A}}$} & \multirow{2}{*}{$\frac{\mathrm{P} 1000}{2.89 \mathrm{~B}}$} & \multirow{2}{*}{$\frac{S Y}{14.3 C}$} \\
\hline \multirow{4}{*}{$\mathrm{N}$} & 60 & & & & & & & & \\
\hline & 90 & $38.1 \mathrm{C}$ & 267.6B & $3.70 A B$ & $19.7 C$ & $31.9 A B$ & $250.8 \mathrm{~A}$ & $3.13 A B$ & $15.5 \mathrm{~B}$ \\
\hline & 120 & $40.2 \mathrm{~A}$ & $270.4 \mathrm{~A}$ & $3.90 \mathrm{~A}$ & $23.0 \mathrm{~A}$ & $32.7 \mathrm{AB}$ & $251.4 \mathrm{~A}$ & $3.35 \mathrm{AB}$ & $16.3 \mathrm{~B}$ \\
\hline & 150 & $39.1 \mathrm{~B}$ & 268.9AB & $3.84 \mathrm{~A}$ & $21.3 \mathrm{~B}$ & $33.4 \mathrm{~A}$ & $253.0 \mathrm{~A}$ & $3.58 \mathrm{~A}$ & $17.9 \mathrm{~A}$ \\
\hline \multirow{4}{*}{ K } & 60 & $37.7 \mathrm{~B}$ & 266.0B & $3.63 \mathrm{~A}$ & $17.5 \mathrm{D}$ & $30.7 \mathrm{C}$ & $247.2 \mathrm{~A}$ & $2.99 \mathrm{~A}$ & $14.4 \mathrm{C}$ \\
\hline & 90 & $38.2 \mathrm{~B}$ & 267.5B & $3.65 \mathrm{~A}$ & $19.5 \mathrm{C}$ & $31.5 \mathrm{BC}$ & $249.8 \mathrm{~A}$ & $3.19 \mathrm{~A}$ & $15.8 B$ \\
\hline & 120 & $39.3 \mathrm{~A}$ & $269.4 \mathrm{~A}$ & $3.74 \mathrm{~A}$ & $21.9 \mathrm{~B}$ & $32.9 \mathrm{AB}$ & $252.3 \mathrm{~A}$ & $3.33 \mathrm{~A}$ & 16.1B \\
\hline & 150 & $39.8 \mathrm{~A}$ & $271.4 \mathrm{~A}$ & $3.84 \mathrm{~A}$ & $22.9 \mathrm{~A}$ & $33.9 \mathrm{~A}$ & $255.3 \mathrm{~A}$ & $3.43 \mathrm{~A}$ & $17.7 \mathrm{~A}$ \\
\hline \multirow{16}{*}{$\mathrm{N}: \mathrm{K}$} & $60: 60$ & $35.2 f$ & $265.7 b-d$ & $3.23 \mathrm{~B}$ & $15.2 f$ & $29.9 b$ & $246.6 a$ & $2.86 a$ & $13.7 f$ \\
\hline & $60: 90$ & $38.5 b-d$ & $265.7 \mathrm{~cd}$ & 3.39ab & $17.5 \mathrm{e}$ & $30.4 b$ & $247.5 a$ & $2.81 a$ & 14.2ef \\
\hline & $60: 120$ & $38.2 \mathrm{c}-\mathrm{e}$ & 267.4a-d & $3.59 \mathrm{ab}$ & 18.8ed & $32.0 \mathrm{ab}$ & $249.3 a$ & $2.98 a$ & $14.4 \mathrm{ef}$ \\
\hline & $60: 150$ & $38.2 \mathrm{c}-\mathrm{e}$ & $270.7 a-c$ & $3.45 a b$ & $19.4 d$ & $31.9 a b$ & $254.7 a$ & $2.92 a$ & $14.9 \mathrm{~d}-\mathrm{f}$ \\
\hline & $90: 60$ & $38.5 b-d$ & $262.3 d$ & $4.05 a$ & $15.2 f$ & $30.3 b$ & $249.6 a$ & $2.72 a$ & $14.3 \mathrm{ef}$ \\
\hline & $90: 90$ & $36.1 \mathrm{ef}$ & 268.3a-c & $3.56 \mathrm{ab}$ & $17.3 e$ & $30.1 b$ & $248.7 a$ & $3.09 a$ & $15.4 \mathrm{~d}-\mathrm{f}$ \\
\hline & $90: 120$ & $38.9 a-d$ & 269.3a-c & $3.42 \mathrm{ab}$ & $22.4 b c$ & $33.3 a b$ & $250.3 a$ & $3.29 a$ & $15.5 c-f$ \\
\hline & $90: 150$ & $39.1 b-d$ & $270.6 a-c$ & $3.76 \mathrm{ab}$ & 23.9ab & $33.9 a b$ & $254.5 a$ & $3.42 a$ & $16.8 b-e$ \\
\hline & $120: 60$ & $39.7 a-c$ & 269.1a-c & $3.80 \mathrm{ab}$ & 22.4ab & $32.1 \mathrm{ab}$ & $248.3 a$ & $3.00 \mathrm{a}$ & $14.4 \mathrm{ef}$ \\
\hline & $120: 90$ & $40.0 a-c$ & 268.8a-c & $3.85 a b$ & $22.6 a b$ & $32.0 \mathrm{ab}$ & $249.0 a$ & $3.28 a$ & $15.6 c-f$ \\
\hline & $120: 120$ & $39.8 a-c$ & $271.4 a b$ & $3.78 a b$ & $23.0 \mathrm{ab}$ & $32.6 a b$ & $253.6 a$ & $3.50 \mathrm{a}$ & $16.0 \mathrm{~b}-\mathrm{f}$ \\
\hline & $120: 150$ & $41.5 a$ & $272.3 a$ & $4.15 a$ & $24.0 a$ & $34.1 \mathrm{ab}$ & $254.8 a$ & $3.60 a$ & 19.0ab \\
\hline & $150: 60$ & $37.4 d-f$ & 267.1a-d & $3.43 a b$ & $17.3 e$ & $30.7 b$ & $244.4 a$ & $3.37 a$ & $15.1 d-f$ \\
\hline & $150: 90$ & $38.1 \mathrm{c}-\mathrm{e}$ & 267.2a-d & $3.79 a b$ & $20.5 \mathrm{~cd}$ & $33.7 a b$ & $254.2 a$ & $3.59 \mathrm{a}$ & 18.0a-d \\
\hline & $150: 120$ & $40.3 a-c$ & $269.4 a-c$ & $4.14 a$ & 23.3ab & $33.6 a b$ & $256.1 a$ & $3.57 a$ & 18.6a-c \\
\hline & $150: 150$ & $40.6 a b$ & $272.1 \mathrm{a}$ & $3.98 \mathrm{ab}$ & $24.2 a$ & $35.6 a$ & $257.3 a$ & $3.79 a$ & $20.0 a$ \\
\hline \multicolumn{10}{|c|}{ Analysis of variance } \\
\hline & $N$ & $\star \star *$ & ** & ** & $* * *$ & ** & ** & ** & *** \\
\hline & $\mathrm{K}$ & $* * *$ & $* * *$ & ns & $* * *$ & $* * *$ & Ns & ns & *** \\
\hline & $x \mathrm{~K}$ & *** & ns & * & *** & Ns & Ns & ns & * \\
\hline
\end{tabular}

Different capital letters show significance among $\mathrm{N}$, and $\mathrm{K}$ treatments, different lowercase letters show significance among $\mathrm{N}$ combined $\mathrm{K}$ treatments at $\mathrm{p}<0.05$ by Turkey's test. $\mathrm{ns}=$ not significant; $*=$ significant at $0.05 ; * *=$ significant at $0.01 ; * * *=$ significant at 0.001 .

\section{Effect of nitrogen and potassium on seed nutrient of quinoa}

$\mathrm{N}$ application had significant effect on the protein and starch contents in both locations, the fiber content in Dak Lak, lipid content in Dak Nong. Meanwhile, the K application had significant effect on the protein content in both locations, fiber content in Dak Lak, and starch content in Dak Nong. Both $\mathrm{N}$ and $\mathrm{K}$ had no effects on the carbohydrate contents in quinoa seeds. In both locations, the protein content reached peaks when the $\mathrm{N}$ and $\mathrm{K}$ application rates increased to $120 \mathrm{~kg}$ $\mathrm{N} \mathrm{ha}^{-1}$ and $90 \mathrm{~kg} \mathrm{~K}_{2} \mathrm{O} \mathrm{ha}^{-1}$, respectively; after that, the increases were not significant at higher rates. Similarly, the lipid content increased along with the amount of $\mathrm{N}$ and $\mathrm{K}$ application, but a significant difference was found between lower $\mathrm{N}$ treatments (60 and $90 \mathrm{~kg} \mathrm{~N} \mathrm{ha}^{-1}$ ) and $150 \mathrm{~kg} \mathrm{~N} \mathrm{ha}^{-1}$ in Dak Nong only. In contrast, there were downward trends in starch and fiber contents along with the increase of $\mathrm{N}$ and $\mathrm{K}$ application rates. However, the remarkable variation was just found between treatment $60 \mathrm{~kg} \mathrm{~N}^{-1}$ with higher $\mathrm{N}$ treatments (120 and $150 \mathrm{~kg} \mathrm{~N} \mathrm{ha}^{-1}$ ) for starch, and between the treatment of $60 \mathrm{~kg} \mathrm{ha}^{-1}$ of $\mathrm{N}$ and $\mathrm{K}$ with treatments of 90 and $105 \mathrm{~kg} \mathrm{ha}^{-1}$ in Dak Lak. The interaction of $\mathrm{N}$ and $\mathrm{K}$ application was significant 
Table 5. Effects of nitrogen and potassium on contents of protein (Pro.), starch (Sta.), lipid (Lip.), fiber (Fib.), and ash of quinoa in Dak Lak and Dak Nong provinces

\begin{tabular}{|c|c|c|c|c|c|c|c|c|c|c|c|}
\hline \multirow{2}{*}{\multicolumn{2}{|c|}{ Treatment }} & \multicolumn{5}{|c|}{ Dak Lak } & \multicolumn{5}{|c|}{ Dak Nong } \\
\hline & & \multirow{2}{*}{$\frac{\text { Pro. }}{18.0 C}$} & \multirow{2}{*}{$\begin{array}{c}\text { Sta. } \\
62.8 \mathrm{~A}\end{array}$} & \multirow{2}{*}{$\frac{\text { Lip. }}{4.7 A}$} & \multirow{2}{*}{$\begin{array}{l}\text { Fib. } \\
6.0 \mathrm{~A}\end{array}$} & \multirow{2}{*}{$\begin{array}{l}\text { Ash } \\
4.7 \mathrm{~A}\end{array}$} & \multirow{2}{*}{$\begin{array}{c}\text { Pro. } \\
17.2 \mathrm{C}\end{array}$} & \multirow{2}{*}{$\begin{array}{c}\text { Sta. } \\
64.2 \mathrm{~A}\end{array}$} & \multirow{2}{*}{$\frac{\text { Lip. }}{\text { 4.9B }}$} & \multirow{2}{*}{$\begin{array}{l}\text { Fib. } \\
7.2 \mathrm{~A}\end{array}$} & \multirow{2}{*}{$\frac{\text { Ash }}{3.9 \mathrm{~A}}$} \\
\hline \multirow{4}{*}{$\mathrm{N}$} & 60 & & & & & & & & & & \\
\hline & 90 & 19.4BC & $61.8 A B$ & $4.9 \mathrm{~A}$ & $5.7 \mathrm{AB}$ & $4.7 \mathrm{~A}$ & $18.4 \mathrm{BC}$ & $62.4 \mathrm{~A}$ & $5.0 \mathrm{~B}$ & $7.6 \mathrm{~A}$ & $3.6 \mathrm{~A}$ \\
\hline & 120 & $22.08 \mathrm{~A}$ & $60.2 \mathrm{BC}$ & $5.5 \mathrm{~A}$ & $4.8 \mathrm{~B}$ & $4.7 \mathrm{~A}$ & $19.5 \mathrm{AB}$ & $59.9 \mathrm{~B}$ & $5.4 \mathrm{AB}$ & $7.7 \mathrm{~A}$ & $3.7 \mathrm{~A}$ \\
\hline & 150 & $20.7 \mathrm{AB}$ & $60.0 \mathrm{C}$ & $5.4 \mathrm{~A}$ & $4.7 \mathrm{~B}$ & $4.7 \mathrm{~A}$ & $20.9 \mathrm{~A}$ & $59.2 B$ & $5.7 \mathrm{~A}$ & $7.3 \mathrm{~A}$ & $3.8 \mathrm{~A}$ \\
\hline \multirow{4}{*}{$\mathrm{K}$} & 60 & $18.0 \mathrm{~B}$ & $62.0 \mathrm{~A}$ & $5.2 \mathrm{~A}$ & $5.9 \mathrm{~A}$ & $4.8 \mathrm{~A}$ & $17.2 \mathrm{C}$ & $62.9 \mathrm{~A}$ & $5.2 \mathrm{~A}$ & $7.3 \mathrm{~A}$ & $3.8 \mathrm{~A}$ \\
\hline & 90 & $19.4 \mathrm{~B}$ & $61.3 \mathrm{~A}$ & $5.3 \mathrm{~A}$ & $5.4 \mathrm{AB}$ & $4.6 \mathrm{~A}$ & $18.5 \mathrm{BC}$ & $62.3 \mathrm{~A}$ & $5.2 \mathrm{~A}$ & $7.4 \mathrm{~A}$ & $3.6 \mathrm{~A}$ \\
\hline & 120 & $20.9 \mathrm{~A}$ & $60.9 \mathrm{~A}$ & $5.4 \mathrm{~A}$ & $5.1 \mathrm{~B}$ & $4.7 \mathrm{~A}$ & $19.3 \mathrm{AB}$ & $61.2 \mathrm{~A}$ & $5.3 \mathrm{~A}$ & $7.5 \mathrm{~A}$ & $3.7 \mathrm{~A}$ \\
\hline & 150 & $21.8 \mathrm{~A}$ & $60.6 \mathrm{~A}$ & $4.5 \mathrm{~A}$ & $4.7 \mathrm{~B}$ & $4.7 \mathrm{~A}$ & $21.0 \mathrm{~A}$ & $59.3 \mathrm{~B}$ & $5.4 \mathrm{~A}$ & $7.7 \mathrm{~A}$ & $3.9 \mathrm{~A}$ \\
\hline \multirow{16}{*}{$\mathrm{N}: \mathrm{K}$} & $60: 60$ & $18.1 \mathrm{de}$ & $63.9 a$ & $4.1 \mathrm{a}$ & $6.6 a$ & $4.6 a$ & $14.6 \mathrm{e}$ & $67.1 \mathrm{a}$ & $4.4 a b$ & $6.6 a$ & $4.0 \mathrm{a}$ \\
\hline & $60: 90$ & 18.3de & $62.3 a b$ & $4.4 a$ & $6.5 a$ & $4.9 a$ & $16.5 \mathrm{de}$ & $66.2 a$ & $4.3 b$ & $7.1 \mathrm{a}$ & $3.6 a$ \\
\hline & $60: 120$ & $16.5 \mathrm{e}$ & $62.8 a b$ & $6.0 a$ & $5.8 a$ & $4.9 a$ & $17.2 \mathrm{~b}-\mathrm{e}$ & $64.7 \mathrm{ab}$ & $4.8 a b$ & $7.2 a$ & $3.9 a$ \\
\hline & $60: 150$ & $19.1 \mathrm{c}-\mathrm{e}$ & $62.3 a b$ & $4.2 \mathrm{a}$ & $5.3 a$ & $4.4 a$ & 20.3a-d & $58.9 \mathrm{~cd}$ & 6.0ab & $7.9 a$ & $4.2 a$ \\
\hline & $90: 60$ & $16.7 e$ & 63.1ab & $5.3 a$ & $6.2 \mathrm{a}$ & $4.8 a$ & $16.3 \mathrm{de}$ & 64.7ab & $5.1 \mathrm{ab}$ & $7.4 a$ & $3.7 a$ \\
\hline & $90: 90$ & $20.1 \mathrm{~b}-\mathrm{e}$ & $61.9 a b$ & $5.1 a$ & $5.4 a$ & $4.4 a$ & $17.1 \mathrm{c}-\mathrm{e}$ & 63.9a-c & $5.0 a b$ & $7.4 a$ & $3.6 a$ \\
\hline & $90: 120$ & $21.9 a-d$ & $61.1 \mathrm{ab}$ & $4.2 \mathrm{a}$ & $4.7 \mathrm{a}$ & $4.5 a$ & $18.7 a-e$ & $60.8 b-d$ & $5.1 a b$ & $8.2 \mathrm{a}$ & $3.4 a$ \\
\hline & $90: 150$ & $19.0 \mathrm{de}$ & 61.1ab & $4.8 a$ & $6.5 a$ & $5.1 a$ & $21.4 a-c$ & $60.2 b-d$ & $4.9 a b$ & $7.6 a$ & $3.5 a$ \\
\hline & $120: 60$ & $19.5 b-e$ & $60.6 a b$ & $5.3 a$ & $5.9 a$ & $5.1 a$ & 18.9a-e & $60.8 b-d$ & $5.1 a b$ & $7.1 a$ & $3.5 a$ \\
\hline & $120: 90$ & $20.7 b-e$ & $60.2 \mathrm{ab}$ & $5.9 a$ & $4.9 a$ & $4.9 a$ & 19.6a-d & $59.6 b-d$ & $5.4 a b$ & $7.6 a$ & $3.6 a$ \\
\hline & $120: 120$ & $22.3 a-d$ & $60.2 \mathrm{ab}$ & $5.8 a$ & $4.1 \mathrm{a}$ & $4.9 a$ & 19.3a-e & $59.7 b-d$ & $5.7 a$ & $7.6 a$ & $3.9 a$ \\
\hline & $120: 150$ & $25.9 a$ & $58.9 b$ & $4.9 a$ & $4.1 \mathrm{a}$ & $4.0 \mathrm{a}$ & 20.3a-d & $59.7 \mathrm{~b}-\mathrm{d}$ & $5.3 a b$ & $8.3 a$ & $4.0 \mathrm{a}$ \\
\hline & $150: 60$ & $17.8 \mathrm{de}$ & $60.2 \mathrm{ab}$ & $6.0 a$ & $5.1 a$ & $4.7 a$ & 18.9a-e & $59.1 \mathrm{~cd}$ & $6.2 \mathrm{a}$ & $8.0 a$ & $3.9 a$ \\
\hline & $150: 90$ & $18.5 \mathrm{de}$ & $61.0 \mathrm{ab}$ & $5.8 a$ & $4.9 a$ & $4.4 a$ & $20.9 a-d$ & $59.4 \mathrm{~cd}$ & $5.9 a b$ & $7.4 a$ & $3.5 a$ \\
\hline & $150: 120$ & 23.1a-c & 59.3ab & $5.5 a$ & $4.2 \mathrm{a}$ & 4.3a & $21.9 a b$ & $59.8 b-d$ & $5.4 a b$ & $6.9 a$ & $3.4 a$ \\
\hline & $150: 150$ & $23.4 a b$ & $60.3 a b$ & $4.1 \mathrm{a}$ & $4.6 a$ & $5.3 a$ & $22.0 a$ & $58.4 d$ & $5.3 a b$ & $6.9 a$ & $3.8 a$ \\
\hline \multicolumn{12}{|c|}{ Analysis of anova } \\
\hline & $\mathrm{N}$ & $* * *$ & *** & ns & ** & ns & $* * *$ & *** & * & ns & $\mathrm{ns}$ \\
\hline & K & *** & ns & ns & * & ns & $* \star *$ & *** & ns & ns & ns \\
\hline & $\mathrm{JK}$ & *** & ns & ns & ns & ns & ns & Ns & ns & ns & ns \\
\hline
\end{tabular}

Different capital letters show significance among N, and K treatments, different lowercase letters show significance among $\mathrm{N}$ combined $\mathrm{K}$ treatments at $\mathrm{p}<0.05$ by Turkey's test. $\mathrm{ns}=$ not significant; $*=$ significant at $0.05 ; * *=$ significant at $0.01 ; * * *=$ significant at 0.001 .

for the protein content in both locations, the better values resulted from the combinations of $\mathrm{K}$ at the rate of $105 \mathrm{~kg} \mathrm{ha}^{-1}$ with higher $\mathrm{N}$ application rates.

\section{DISCUSSION}

There was a positive correlation between $\mathrm{N}$ application and quinoa yield with an increase of $\mathrm{N}$ between 0 to $120 \mathrm{~kg} \mathrm{ha}^{-1}$ (Kaul et al. 2005). Under temperate climatic conditions in Denmark, although there was a significant yield increase when the amount of $\mathrm{N}$ fertilizer was raised from 40 to $160 \mathrm{~kg} \mathrm{ha}^{-1}$, quinoa seems to be well adapted to poor soil (Jacobsen et al., 1994). Similarly, increasing $\mathrm{N}$ from 0 to $180 \mathrm{~kg} \mathrm{~N} \mathrm{ha}^{-1}$ resulted in better values for plant height, leaf area, number of seeds per cluster, and total seed yield in quinoa (Al-asadi et al., 2021). Increase $\mathrm{N}$ from 120 to 180 $\mathrm{kg} \mathrm{ha}^{-1}$ enhanced plant height, leaf area, plant dry weight, panicle length, and grain yield of quinoa (Owji et al., 2020). Higher $\mathrm{N}$ application rate (240 $\mathrm{kg} \mathrm{ha}^{-1}$ ) showed better values for plant height, the number of branches, 1000-seed weight, biomass, and seed yield of quinoa in Ras Sadersinai (Fawy et al., 2017). Under sandy soil conditions in Egypt, Shams (2012) revealed that fertilizing quinoa with $360 \mathrm{~kg} \mathrm{~N} \mathrm{ha}^{-1}$ resulted in maximum plant 
height, grain yield, and biological yield. From the obtained study results, the positive impacts of $\mathrm{N}$ and $\mathrm{K}$ application on the growth and yield of quinoa, but after an optimum dose, growth and yield were not significantly changed even decreased when the dose continues increasing. The result is similar to the previous studies. Wang et al. (2020) reported that applying $\mathrm{N}$ at the rate of $240 \mathrm{~kg} \mathrm{ha}^{-1}$ had a significantly greater 1000 -seed weight than 80 and $160 \mathrm{~kg} \mathrm{ha}^{-1}$. Moreover, plant height, leaf area index, dry matter, 1000-seed weight all increased along with $\mathrm{N}$, whereas seed yield did not further increase when the $\mathrm{N}$ rate was beyond 160 $\mathrm{kg} \mathrm{ha}^{-1}$. Under Mediterranean climatic conditions, among seven $\mathrm{N}$ levels ranging from 0 to $175 \mathrm{~kg}$ $\mathrm{ha}^{-1}$, the level of $150 \mathrm{~kg} \mathrm{ha}^{-1}$ was proven to be the best level for $\mathrm{N}$ supplementation for grain yield of quinoa (Geren, 2015). Basra (2014) reported that soil application of $\mathrm{N}$ at $75 \mathrm{~kg} \mathrm{ha}^{-1}$ and higher improved plant height, stem diameter, main panicle length, yield components, but $75 \mathrm{~kg} \mathrm{ha}^{-1}$ was found to be the best level of $\mathrm{N}$ to attain maximum economic harvest in quinoa. In the red river delta in Vietnam, under normal and saline stress conditions of an alluvium soil, the growth parameters, and yield components increased according to the increase of the $\mathrm{N}$ application rates from 0 to 90 $\mathrm{kg} \mathrm{N} \mathrm{ha}{ }^{-1}$, then decreased when the nitrogen rates were higher (Dinh et al., 2015, 2021). Conducting a green-house experiment, Rêgo et al. (2017) found linear correlations of $\mathrm{K}$ application rate with the shoot and root dry mass, but polynomial correlations with the number of grain and grain production in quinoa. The grain yield of quinoa increased significantly when the $\mathrm{K}$ application rate was raised up to $120 \mathrm{~kg} \mathrm{ha}^{-1}$, then slightly decreased by the application at the rate of $180 \mathrm{~kg}$ $\mathrm{ha}^{-1}$ (Salim et al., 2019). Turcios et al. (2020) reported that an adequate supply of $\mathrm{K}$ promoted the growth of quinoa for biomass and leaf area under both non-stress and saline-stress conditions.

In a recent study, higher levels of $\mathrm{N}$ and $\mathrm{K}$ application resulted in greater protein and fat content, but lower starch and fiber contents, compared to lower levels. Abou-Amer and Kamel (2011), Wang et al. (2020) agreed that the protein content in quinoa seeds further increased with a higher N rate. Geren (2015) also found upward trends in crude protein content in quinoa seed by increasing $\mathrm{N}$ application rates from 0 to 175 $\mathrm{kg} \mathrm{ha}^{-1}$. Similarly, Kakabouki et al. (2014) stated that crude protein content was higher in the treatment of $200 \mathrm{~kg} \mathrm{~N} \mathrm{ha}^{-1}$ compared to $100 \mathrm{~kg} \mathrm{~N}$ ha $^{-1}$. Gomaa et al. (2013) revealed that the crude protein percentage in quinoa seed increased as a result of the increasing rate of ammonium nitrate. Almadini et al. (2019) reported that increased $\mathrm{N}$ rate from 0 to $160 \mathrm{~kg} \mathrm{~N}^{-1}$ promoted the contents of protein, fat, starch, and ash. Thanapornpoonpong (2004) agreed that the protein and starch contents increased, but the fat content decreased by increasing $\mathrm{N}$ levels.

Fertilizer supplements should be practiced to satisfy the crop nutrient requirement. In quinoa, Moreale (1993) recorded that producing one ton of stover plant requires $5.0,1.8$, and $32.5 \mathrm{~kg} \mathrm{~N}, \mathrm{P}$, and $\mathrm{K}$, respectively, and to produce seed yield of about 4 ton ha ${ }^{-1}$, quinoa uptake $95 \mathrm{~kg} \mathrm{~N}, 27 \mathrm{~kg} \mathrm{P}$, and $185 \mathrm{~kg} \mathrm{~K}$. Therefore, supply of 100 to $150 \mathrm{~kg}$ $\mathrm{N} \mathrm{ha}^{-1}, 30 \mathrm{~kg} \mathrm{P}$ (corresponding to $70 \mathrm{~kg} \mathrm{P}_{2} \mathrm{O}_{5}$ ) and 100 to $200 \mathrm{~kg} \mathrm{~K}$ (corresponding to 125 to $250 \mathrm{~kg}$ $\mathrm{K}_{2} \mathrm{O}$ ) is recommended in Netherland and Demark. Alvar-Beltrán et al. (2021) reported that while $\mathrm{N}$ and $\mathrm{K}$ are required at medium to fairly high amounts, $\mathrm{P}$ is needed in lower amounts. They also suggested that $12.7,1.6$, and $35.5 \mathrm{~kg} \mathrm{ha}^{-1}$ of $\mathrm{N}, \mathrm{P}$, and $\mathrm{K}$, respectively, should be added into the soil to produce one ton of total biomass (including seeds, stem, and leaves). In this study, with a base dose of $\mathrm{P}$ at $60 \mathrm{~kg} \mathrm{ha}^{-1}$, the combinations of $\mathrm{K}$ at the rate of $150 \mathrm{~kg} \mathrm{ha}^{-1}$ with $\mathrm{N}$ at higher rates $(120$ and $150 \mathrm{~kg} \mathrm{ha}^{-1}$ ) achieved better growth, yield components, and protein contents. Abdolahpour et al. (2020) agreed that the higher amounts of N, $\mathrm{P}$, and $\mathrm{K}$ application showed greater plant height, the number of branches, 1000 -seed weight, seed yield, and protein content in quinoa seed.

In the conducted study, the quinoa grown in ferals soil in Dak Lak seemed to be better in acrisols soil in Dak Nong for growth, yield traits as well as seed quality in terms of protein content. The dry season in Central Highland is often from October to May. In this study, quinoa was grown for half end of the dry season and rainfall came sooner in Dak Lak. Total rainfall during quinoa growing in this location was also 2.5 times higher than that in Dak Nong. However, rainfall seemed to be not the main factor that affected the growth and yield in this region. In the same location in Dak Lak province, Nguyen et al. (2020) observed that compensating for lower plant height and panicle numbers, quinoa in the dry season had a greater panicle length, seed number per panicle, 1000-seed weight, and seed yield compared to it in the rainy season. The reason may be from soil fertility. The ferralsols soil is the most fertile soil, whereas acrisols 
soil is a poor fertility one in the Central Highland. Richer soil nutrients may lead the plant to reach the saturation points for growth, yield, and quality sooner. In fact, plant diameter, the number of branches, yield components, and protein content of quinoa in Dak Lak decreased when the supply of $\mathrm{N}$ was over the optimum dose of $120 \mathrm{~kg} \mathrm{~N} \mathrm{ha}^{-1}$ in Dak Lak but continuously increased by raising $\mathrm{N}$ application from 60 to $150 \mathrm{~kg} \mathrm{ha}^{-1}$. Kansomjet et al. (2017) also found greater growth and yield of two quinoa varieties Moradas and Verdes in Pang-Da which had richer soil fertility, compared to Phabadhuaytom. The highest seed yield and 1000-seed weight were achieved at a rate of $93.75 \mathrm{~kg} \mathrm{~N} \mathrm{ha}^{-1}$ in Pang-Da and $187.50 \mathrm{~kg} \mathrm{~N}$ ha $^{-1}$ in Phabadhuaytom, respectively.

\section{CONCLUSIONS}

The resulted showed positive effects of $\mathrm{N}$ and $\mathrm{K}$ application on growth, yield, and seed nutrients. It was also shown that the quinoa grown in ferrasols soil in Dak Lak seemed to be better in acrisols soil in Dak Nong for growth, yield traits as well as seed quality in terms of protein content. The optimum doses to suggest for quinoa culture should be based on soil fertility. In terms of ferralsols and acrisols soils in Central Highland, the recommended dose of fertilizer was $150 \mathrm{~kg} \mathrm{~N}, 60$ $\mathrm{kg} \mathrm{P}_{2} \mathrm{O}_{5}$, and $105 \mathrm{~kg} \mathrm{~K}_{2} \mathrm{O}$ per hectare.

\section{Acknowledgments}

The quinoa seeds were provided by Dr. Robert Van Loo, Wageningen Plant Research, Netherland. We are grateful for the financial support throughout the research project B2020-TTN-03 "Study on quinoa varietal selection and cultivation technique for quinoa (Chenopodium quinoa Willd.) in Central Highland", which was funded by the Ministry of Education and Training, Vietnam from 2020-2021.

\section{REFERENCES}

1. Abdolahpour H., Nejad E.T., Pour A.P. 2021. Effect of nitrogen, phosphorus and potassium fertilizers on morpho-physiological characteristics and seed yield of quinoa (Chenopodium quinoa Willd.). Journal of Crop Ecophysiology, 15(1). DOI: 10.30495/ jcep.2021.681006

2. Abou-Amer A.I., Kamel A.S. 2021. Growth, yield and nitrogen utilization efficiency of quinoa (Chenopodium quinoa) under different rates and methods of nitrogen fertilization. Egyptian Journal of Agronomy, 33(2), 155-166.

3. Almadini A.M., Badran A.E., Algosaibi A.M. 2019. Evaluation of efficiency and response of quinoa plant to nitrogen fertilization levels. Middle East Journal of Applied Sciences, 9(4), 839-849.

4. Alvar-Beltrán J., Napoli M., Dao A., Amoro O., Verdi L., Orlandini S., Marta A.D. 2021. Nitrogen, phosphorus and potassium mass balances in an irrigated quinoa field. Italian Journal of Agronomy, 16, 1788. DOI: 10.481/ija.2021.1788.

5. Al-asadi W.A.J,Al-dogagy K.A. 2021. The effect of nitrogen fertilizer levels and planting dates on some growth traits and yield of the quinoa plant (Chenopodium quinoa Wild). Al-Muthanna Journal for Agricultural Sciences. DOI: 10.52113/mjas04/8.2/27

6. Basra S.M.A, Iqbal S., Afzal I. 2014. Evaluating the response of nitrogen application on growth, development and yield of quinoa genotypes. International Journal of Agriculture and Biology, 16(5), 886-892.

7. Bertero H.D., Vega A.J.D.L, Correa G., Jacobsen S.E., Mujica A. 2004. Genotype and genotype-byenvironment interaction effects for grain yield and grain size of quinoa (Chenopodium quinoa Willd) as revealed by pattern analysis of international multi-environment trials. Field Crop Research, 89, 299-318.

8. Dinh T.H., Nguyen T.C., Nguyen V.L. 2015. Effect of nitrogen on growth and yield of quinoa accessions. Journal of Science and Development, 13(2), 173-182.

9. Dinh T.H., Dang T.P.A., Luu H.N., Nguyen V.L. 2021. Effects of nitrogen application on the growth and yield of quinoa under saline conditions in Northern Vietnam. Vietnam Journal of Agricultural Sciences, 4(1), 903-911. DOI: 10.31817/10.31817/ vjas.2021.4.1.01.

10. Eckstein D., Künzel V., Schäfer L. 2017. Globl climate risk index 2018. Germanwatch. https://germanwatch.org/en/node/14987.

11. Eisa S.S., Abd El-Samd E.H., Hussin S.A., Ali E.A., Ebrahim M., González J.A., Ordano M. Erazzú L.E., El-Bordeny N.E., Abdel-Ati A.A. 2018. Quinoa in Egypt - Plant density effects on seed yield and nutrional quality in Marginal regions. Middle East Journal of Applied Sciences, 8(2), 515-522.

12. FAO. 2011. Quinoa: An ancient crop to contribute to world food security. Regional Office for Latin America and the Caribbean. http://www.cnafun.moa.gov. cn/zl/tstpzl/201305/P020130509357618775387.pdf.

13. Fawy H.A., Attia M.F., Hagab R.H. 2017. Effect of nitrogen fertilization and organic acids on grains productivity and biochemical contents of quinoa plant grown under soil conditions of Ras Sadersinai. Egyptian Journal of Desert Research, 67(1), 171-185.

14. Geren H. 2015. Effects of different nitrogen levels on the grain yield and some yield components of quinoa (Chenopodium quinoa willd.) under 
Mediterranean climatic conditions. Turkish Journal of Field Crops, 20, 59-64. DOI: 10.17557/.39586.

15. Gomaa E.F. 2013. Effect of nitrogen, phosphorus and biofertilizers on quinoa plant. Journal of Applied Sciences Research, 9(8), 5210-5222.

16. Hou W.F., Yan J.Y., Jákli B., Lu J.W., Ren T., Cong R.H., Li X.K. 2018. Synergistic effects of nitrogen and potassium on quantitative limitations to photosynthesis in rice (Oryza sativa L.). Journal of Agricultural and Food Chemistry, 66(20), 5125-5132. DOI: $10.1021 /$ acs.jafc. 8 b01135

17. Jacobsen S.E., Jørgensen I., Stølen O. 1994. Cultivation of quinoa (Chenopodium quinoa) under temperate climatic conditions in Denmark. Journal of Agricultural Science, Cambridge, 122, 47-52.

18. Kakabouki I., Bilalis D., Karkanis A., Zervas G., Tsiplakou E., Hela D. 2014. Effects of fertilization and tillage system on growth and crude protein content of quinoa (Chenopodium quinoa Willd.): An alternative forage crop. Emirates Journal of. Food Agriculture, 26(1), 18-24.

19. Kansomjet P., Thobunluepop P., Lertmongkol S., Sarobol E., Kaewsuwan P., Junhaeng P., Pipattanawong N., Iván M.T. 2017. Response of physiological characteristics, seed yield and seed quality of quinoa under difference of nitrogen fertilizer management. American Journal of Plant Physiology, 12(1), 20-27. DOI: 10.3923/ajpp.2017.20.27

20. Kaul H.P., Kruse M., Aufhammer W. 2005. Yield and nitrogen utilization efficiency of the pseudocereals amaranth, quinoa, and buckwheat under differing nitrogen fertilization. European Journal Agronomy, 22(1), 95-100.

21. Moreale A., De Braeckelaer P., Galwey N.W., McNabb J., Meerman, Darwinkel A., Stolen O., Lomholt A., Mastebroek D., Donini B., Haaber J., Steeneken P.A.M. The quinoa project. AIR2.CT93.1426(SC).

22. Nguyen V.L. 2020. Quinoa varietal selection and product development for suitable ecological areas in Vietnam. Ministry of Science and Technology, HNQT/SPĐP/07.17. https://www.most.gov.vn/vn/tintuc/19187/thong-tin-ve-ket-qua-thuc-hien-nhiem-vukhcn-cap-quoc-gia-nghien-cuu-tuyen-chon-va-phattrien-cay-diem-mach-chenopodium-quinoa-wil.aspx.

23. Nguyen V.M., Dinh T.H., Nguyen V.L., Nguyen V.L. 2020. Effects of plant density on growth, yield and seed quality of quinoa genotypes under rainfed conditions on red basalt soil regions. Australian Journal of Crop Science, 14(12), 1977-1982. DOI: 10.21475/ajcs.20.14.12.2849
24. Nguyen V.L., Betero D., Dinh T.H., Nguyen V.L. 2021. Variation in quinoa roots growth responses to drought stresses. Journal of Agronomy and Crop Science. DOI: 10.1111/jac.12528

25. Owji T., Mohajeri F., Madandoust M., Salehi M. 2020. Evaluation of the effect of seed rate and nitrogen fertilizer management on agronomic characteristics and yield components of spring quinoa (Chenopodium quinoa Willd.). International Journal of Pharmaceutical and Phytopharmacological Research, 10(4), 264-272.

26. Rêgo V.M., Koetz M., Bonfim-Silva E.M., da Silva T.J.A., Dourado L.G.A. 2017. Productive characteristics of quinoa (Chenopodium quinoa Willd.) under irrigation and potassium fertilization. Australian Journal of Crop Sciences, 11(11), 1438-1443.

27. Salim S.A., Al-Hadeethi I.K., Alobaydi S.A.J. 2019. Role of irrigation scheduling and potassium fertilization on soil moisture depletion and distributino of quinoa root (irrigation schedulling fertilization and their effect on moisture depletion and yield). Plant Archives, 19(2), 3844-3852.

28. Shams A.S. 2012. Response of quinoa to nitrogen fertilizer rates under sandy soil conditions. Proceeding $13^{\text {th }}$ International Conference of Agronomy, Faculty of Agriculture, Benha University, Egypt, 9-10 September 2012, 195-205.

29. Thanapornpoonpong S. 2004. Effect of nitrogen fertilizer on nitrogen assimilation and seed quality of amaranth (Amaranthus spp.) and quinoa (Chenopodium quinoa Willd). Doctoral dissertation, GeorgAugust-University of Göttingen.

30. Trinh N.D. 2001. Study on develop quinoa (Chenopodium quinoa Willd) in North Vietnam. Doctoral dissertation, Agricultural Academy publishing house, Hanoi, Vietnam.

31. Turcios A., Papenbrock J., Tränkner M. 2021. Potassium, an important element to improve water use efficiency and growth parameters in quinoa (Chenopodium quinoa) under saline conditions. Journal of Agronomy and Crop Science, 207. DOI: $10.1111 /$ jac. 12477

32. Wang N., Wang F., Shock C.C, Meng C., Qiao L. 2020. Effects of management practices on quinoa growth, seed yield, and quality. Agronomy, 10(3), 445. DOI: 10.3390/agronomy 10030445

33. World Bank. 2016. The Vietnam Development Report. Transforming Vietnam agriculture: Gaining more from less. Hong Duc publishing house, Hanoi, April 2016. 